\title{
The objective assessment of contrast sensitivity function by electrophysiological means
}

\author{
J. W. HOWE ${ }^{1}$ AND K. W. MITCHELL ${ }^{2}$
}

From the ${ }^{1}$ Eye Department, University of Newcastle upon Tyne, and the ${ }^{2}$ Regional Department of Medical Physics, Royal Victoria Infirmary, Newcastle upon Tyne NE1 4LP

SUMMARY In recent years it has been shown that it is of considerable clinical value to determine the visual contrast sensitivity function of the patient. This can reveal the wider effects of pathology on the visual system where visual acuity may or may not be affected. There is a comprehensive literature on subjective approaches of obtaining this function in the clinical environment but very little on alternative objective methods. This paper describes the latter approach, which exploits electrophysiological techniques using the visual evoked cortical potential (VECP) to checkerboard onset-offset stimulation. Its application in a variety of disorders of the visual system is described. The importance of selecting the most appropriate stimulus parameters is discussed, and the relative advantages and disadvantages as compared with psychophysical methods are appraised.

In the clinical setting a great deal of reliance is placed on the determination of visual acuity by a Snellen chart. In this test high-contrast optotypes of different angular subtense are viewed under conditions of high (photopic) levels of illumination. Measurement of visual acuity is, however, of limited value, since it determines the resolution limit of the individual only to high-contrast stimuli and does not adequately assess the visual function of the patient. A more complete test of visual performance is to investigate the ability of the subject to resolve stimuli whose contrast varies as well as the angular subtense at the retina. The response so obtained is called the visual contrast sensitivity function (VCSF).

The measurement of this function by sinusoidal luminance profile gratings generated on oscilloscope screens was first described by Campbell and Green. ${ }^{1}$ The contrast of each size of grating is varied until threshold is reached, and a graph of sensitivity ( $1 /$ threshold) versus angular subtense is plotted (Fig. 1). The application of this technique has been well described and reviewed ${ }^{2-28}$ (in some cases with modifications in stimulus form and generation, e.g., TV monitors).

Abnormalities in contrast discrimination can occur in a wide variety of disorders of the visual

Correspondence to Mr. J. W. Howe, FRCS, Eye Department, University of Newcastle upon Tyne, Royal Victoria Infirmary, Newcastle upon Tyne NE1 4LP. system. ${ }^{36-14}$ 16-28 Indeed Arden $^{2930}$ has clearly demonstrated such deficiencies in several ophthalmic diseases in spite of normal visual acuity. A clinical test whereby contrast sensitivity could be determined would therefore provide much additional diagnostic information. Such a test with contrast grating plates was described by Arden and Jacobson ${ }^{31}$ and its

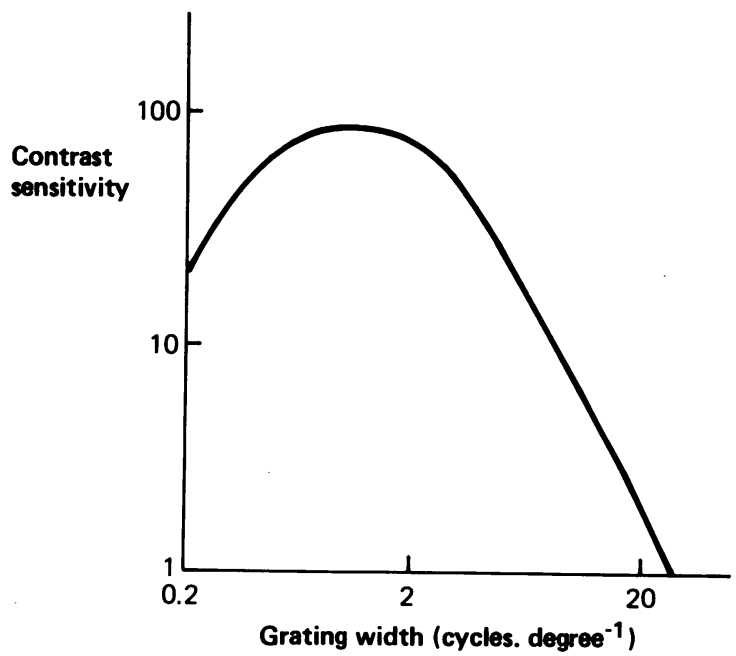

Fig. 1 Psychophysical contrast sensitivity function to sinusoidal luminance profile gratings. 
performance within the clinical environment has been evaluated by several groups. ${ }^{32} 45$

The problem with these techniques is that they are subjective, and certain methodological problems arise in the judgment of threshold level. These drawbacks are exacerbated in the elderly, in children, in confused patients, and in those individuals with disorders which affect higher cognitive processes. A further limitation of such methods is that only threshold data are obtained; no information on suprathreshold function or direct physiological data can be elicited. An objective method of assessing contrast sensitivity which requires only minimal cooperation and no judgment by the patient has obvious advantages. An electrophysiological approach utilising visual evoked cortical potentials (VECPs) fulfils these requirements and has proved possible as a result of the initial work of Campbell and $\mathrm{Maffei}^{46}$ and Campbell and Kulikowski ${ }^{47}$ who demonstrated a good correlation between the so-called 'electrophysiological zero amplitude response' and the psychophysical threshold to temporally modulated sinusoidal gratings.

These techniques have been subsequently developed and refined by means of checkerboard stimulation, with the luminance modulation of the checks designed to produce so-called 'pattern reversal' or 'onset-offset' modes of stimulation. ${ }^{48-57}$ Although the current use of pattern generated VECPs is widespread as an aid to clinical diagnosis, their use in the determination of contrast sensitivity in the clinical environment has not been widely exploited. ${ }^{58-66}$

In this paper we seek to describe a method of obtaining the contrast sensitivity function which we have successfully applied as an aid to routine clinical diagnosis, and which uses fairly standard VECP equipment. This approach does have some problems, but these are common to all evoked potential techniques and can be minimised by careful experimental design and investigation protocol.

\section{Material and methods}

The checkerboard stimulus was produced by a video pattern generator on a high-quality TV monitor. Luminance modulation of the pattern was selected to give the onset-offset mode of stimulation in which the pattern was presented for $40 \mathrm{~ms}$ every $500 \mathrm{~ms}$ (in our case the presentation rate was locked to the $50 \mathrm{~Hz}$ monitor frame rate). The overall luminance of the TV screen-with and without pattern-was maintained constant at $10 \mathrm{Cd} \mathrm{m}^{-2}$. Pattern contrast (defined as $\left(\mathrm{L}_{\max }-\mathrm{L}_{\min }\right) /\left(\mathrm{L}_{\max }+\mathrm{L}_{\min }\right)$ where $\mathrm{L}_{\max }$ and $\mathrm{L}_{\text {min }}$ are the luminance of the bright and dark checks respectively) was altered so that at each check size recordings were made at five or six discrete contrast levels between $2.5 \%$ and $95 \%$ depending on check size. Checks ranging in size from $2 \mathrm{~min}$ to $50 \mathrm{~min}$ subtense were used in the investigations. The TV screen subtended a visual angle of $17^{\circ}$ by $14^{\circ}$ unless otherwise stated.

Silver/silver chloride disc electrodes were attached to the scalp with collodion in the following positions as defined by Jasper ${ }^{67}$ : active $-\mathrm{Oz}$; reference $-\mathrm{Cz}$; earth-Fpz. A Medelec electrophysiological recording unit was used to amplify, average, and store the evoked potentials. The amplifier bandwidth was $0 \cdot 8-$ $80 \mathrm{~Hz}$, and either 64,128 , or 256 epochs of $300 \mathrm{~ms}$ duration were averaged. Two averages were obtained at each contrast level to check for consistency, and amplitude analysis was performed on the average of these two. It was usually found necessary to increase the number of epochs in the average to improve the signal/noise ratio when measuring low-amplitude responses at low contrast levels and/or small check sizes. A permanent record of the responses was made on an X-Y plotter. Amplitude data from the traces were then fed into a MINC PDP-11 computer for storage and analysis.

The subject was instructed to maintain fixation and focus on a small LED marker attached to the centre of the screen. This was checked during the investigation by closed circuit TV monitoring. Before the start of the test the subject was preadapted to the luminance of the blank screen for $5 \mathrm{~min}$. This was the only source of illumination in an otherwise darkened room.

In the normative investigations 12 subjects with no known ocular pathology, full visual fields, and corrected acuities of $6 / 6$ or better were studied. Their ages ranged from 18 to $37 \mathrm{yr}($ mean age $=26)$.

Patients with a variety of ophthalmic disorders were also studied in order to assess the feasibility and value of the test in the clinical setting.

\section{Results}

NORMATIVE INVESTIGATIONS

A characteristic evoked potential generated by the onset-offset stimulus is displayed in Fig. 2. Two clear components, $\mathrm{CI}$ and CII, are observed. These are contrast sensitive components which are thought to be generated on the medial surface of the occipital lobes, in striate and extrastriate cortex respectively. ${ }^{4950} \mathrm{Fig}$. 3 shows the evoked potentials obtained from a normal subject at four check sizes and at different contrast levels. The amplitude of the responses is seen to be a function of contrast, in fact the peak-peak amplitude of components $\mathrm{CI}-\mathrm{CII}$ is proportional to log contrast, this relationship being obeyed up to some saturation value at high mean luminance levels. ${ }^{52}$ The data from Fig. 3 are shown 


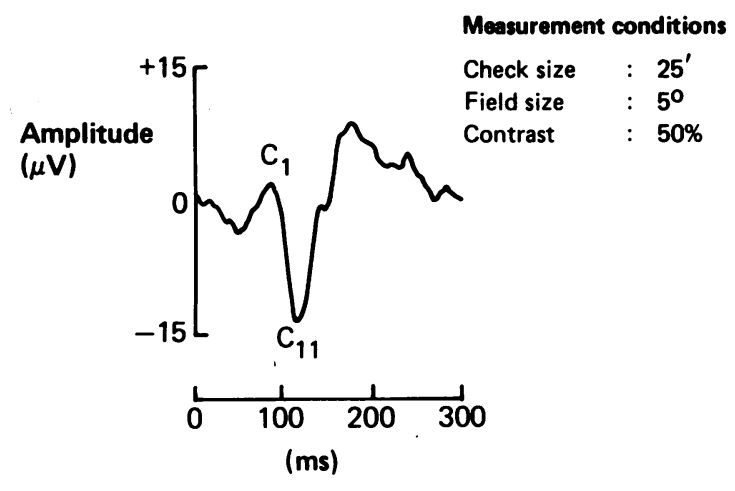

Fig. 2 Normal onset-offset VECP.

plotted in Fig. 4. Least squares regression lines are computed and drawn through the data points and extrapolation to zero amplitude determines the 'electrophysiological contrast threshold' for that particular check size. Confidence limits on these values of threshold are obtained by simple $t$ test analysis. If threshold is then plotted against check subtense, the contrast sensitivity curve is realised (Fig. 5). The shape of the curve is somewhat coarse, as only four points have been plotted. More points are preferable, particularly where it is intended to extrapolate the upper portion of the curve (that portion where sensitivity is falling to smaller checks) to intersect the abscissa at (100\%) contrast (Fig. 6). The significance of this point is that it gives an estimate of the smallest element resolvable by the visual system - that is, a measure of visual acuity. ${ }^{5662}$ When the myope whose data are presented in Fig. 6 had his optical correction removed, the effect on the contrast sensitivity curve is clearly seen; the upper portion of the curve being severely depressed-in keeping with his poorer visual acuity-whereas the lower portion of the curve is not significantly altered.

Since a resolution of $1^{\circ}$ arc is equivalent to an acuity of $6 / 6$ (notwithstanding the fact that perceptual differences exist between checkerboards and Snellen optotypes), then it is possible directly to compare the results with those found by Snellen testing. ${ }^{62}$ In fact the electrophysiological method we use consistently underestimates acuity by about one line on the Snellen chart. The reason for this is that the mean luminance of the TV screen is $1 / 10$ of that of the Snellen chart. The effects of reduced luminance are to produce overall attenuation and a shift of the curve to larger element sizes (Fig. 7) ${ }^{68}$ Therefore visual acuity under these conditions is reduced as a result. This phenomenon is well known in visual system physiology. ${ }^{69}$

In Fig. 8 a mean contrast sensitivity curve for the group of 12 normal individuals is shown plotted.

\section{Pathological STUdies}

Chronic glaucoma. In Fig. 9 the curves from a 58year-old patient who presented with a uniocular raised intraocular pressure are shown. Visual acuities were 6/4 RE, 6/6 LE, but ophthalmoscopy revealed asymmetrical cupping of the optic discs (C:D ratio=

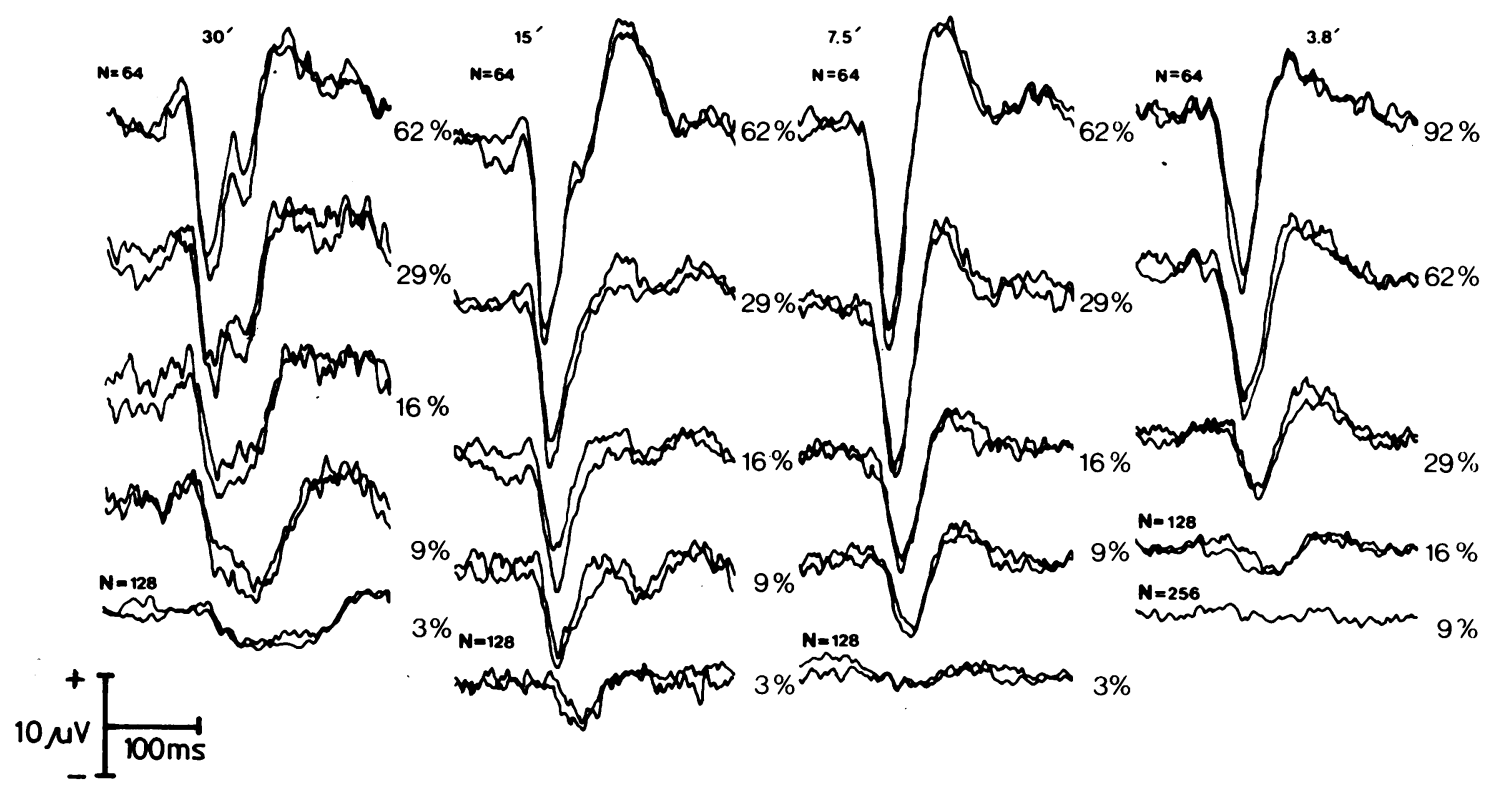

Fig. 3 Onset-offset responses at four different check subtenses and a series of contrasts. 


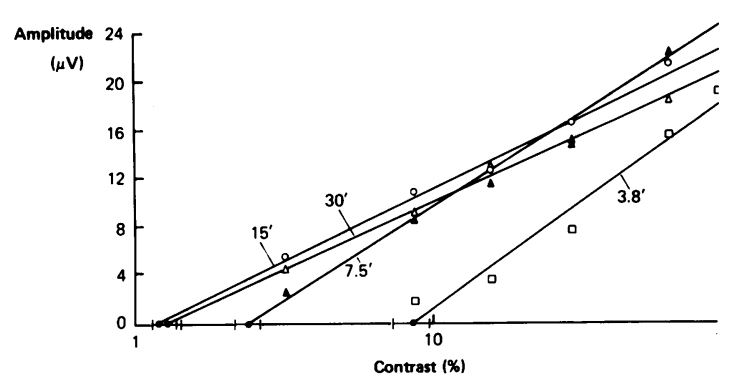

Fig. 4 CI-CII amplitude versus contrast from the data of

Fig. 3.

5:10RE, 7:10LE). Intraocular pressures (IOPs) were $20 \mathrm{mmHg} \mathrm{RE}$ and $32 \mathrm{mmHg}$ LE. Visual fields measured on the Goldmann perimeter showed a full field RE but some vertical enlargement of the blind spot LE. Gonioscopy revealed wide open angles (grade IV). On the basis of these clinical findings a diagnosis of left chronic open-angle glaucoma was made.

The fact that the patient had good Snellen acuity in both eyes correlated well with the observed convergence of the two functions at small check sizes. The divergence in the two curves at larger check sizes, however, represents a significant drop in sensitivity LE. This is what one would generally expect to see in chronic glaucoma as the central retinal fields are classically affected very late in the course of the disease.

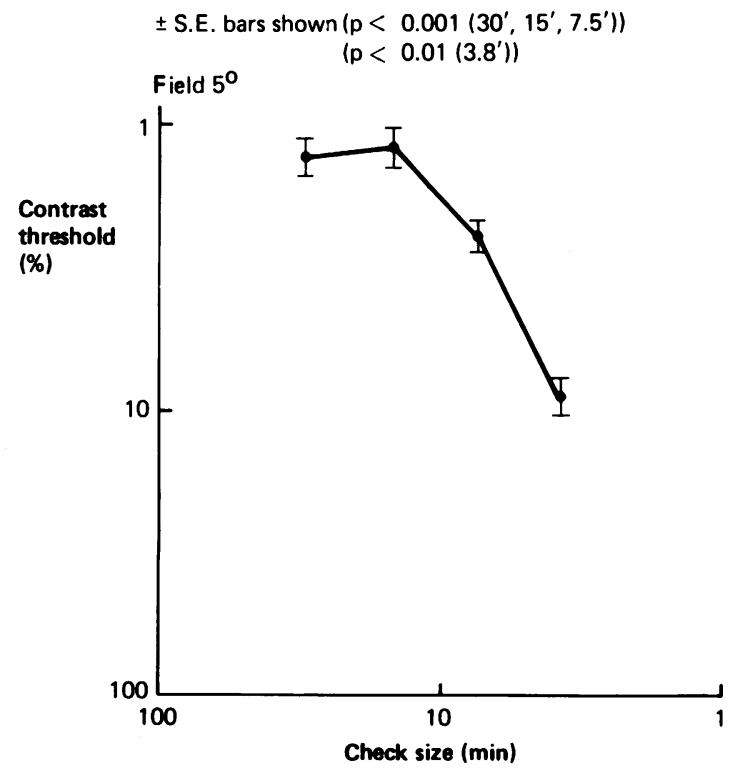

Fig. 5 Electrophysiologically determined contrast sensitivity function obtained from the data of Figs. 3 and 4.

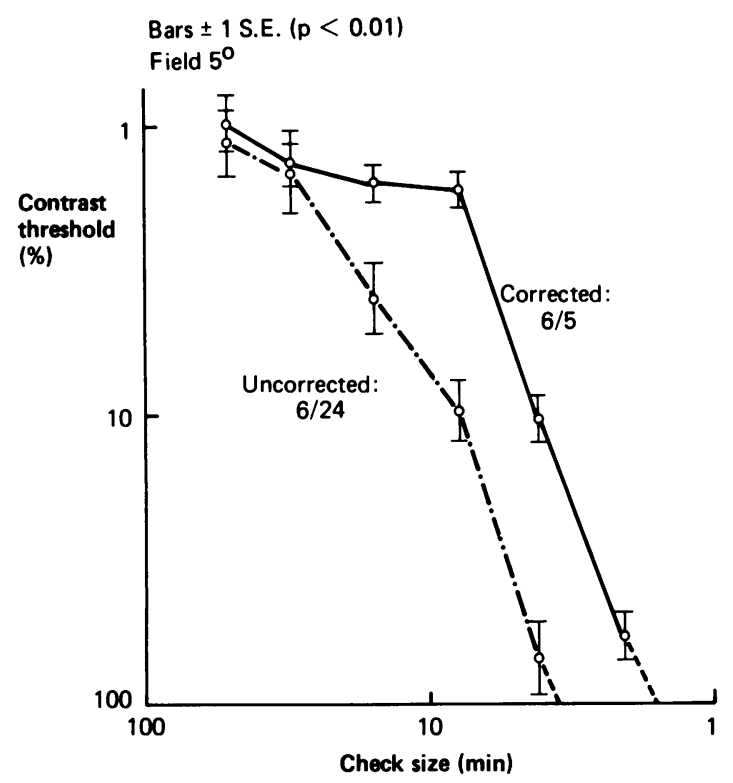

Fig. 6 VCSF of a myope, corrected and uncorrected.

Diabetic retinopathy. Fig. 10 illustrates the contrast sensitivity curve of a 54-year-old diabetic patient with advanced simple retinopathy and left macular oedema. Visual acuities corrected to 6/9 RE and 6/24 LE. The data clearly show a global loss in contrast sensitivity function which correlates well with the patient's subjective loss in acuity and overall form vision.

Macular hole. A 63-year-old patient presented with poor vision $R E$ which she noticed only when the left eye was unintentionally occluded. Visual acuities were $<6 / 60 \mathrm{RE}$ and 6/9 LE. Ophthalmoscopy revealed a macular hole on the right side and this was confirmed by biomicroscopic examination with a Hruby lens. Perimetry revealed an absolute central scotoma of $3^{\circ}$. There were early bilateral lens opacities. The left fundus was entirely healthy and the visual field full. Fig. 11 shows that contrast sensitivity was grossly attenuated to small checks $\left(<10^{\prime}\right.$ subtense). However, the perimacula had survived, and useful form vision to larger stimulus elements was preserved. The VCSF for the intact left eye serves as a useful within-patient comparison, as it can be seen that even this eye had a slight overall sensitivity loss as compared to our normal range. VCSF is known to fall in old age, ${ }^{13343738447071}$ and in addition the patient was experiencing some overall drop in central visual function due to the lens opacities.

Hysterical amblyopia. A 38-year-old patient was referred for examination by his insurance company. $\mathrm{He}$ had sustained a minor head injury at work two 


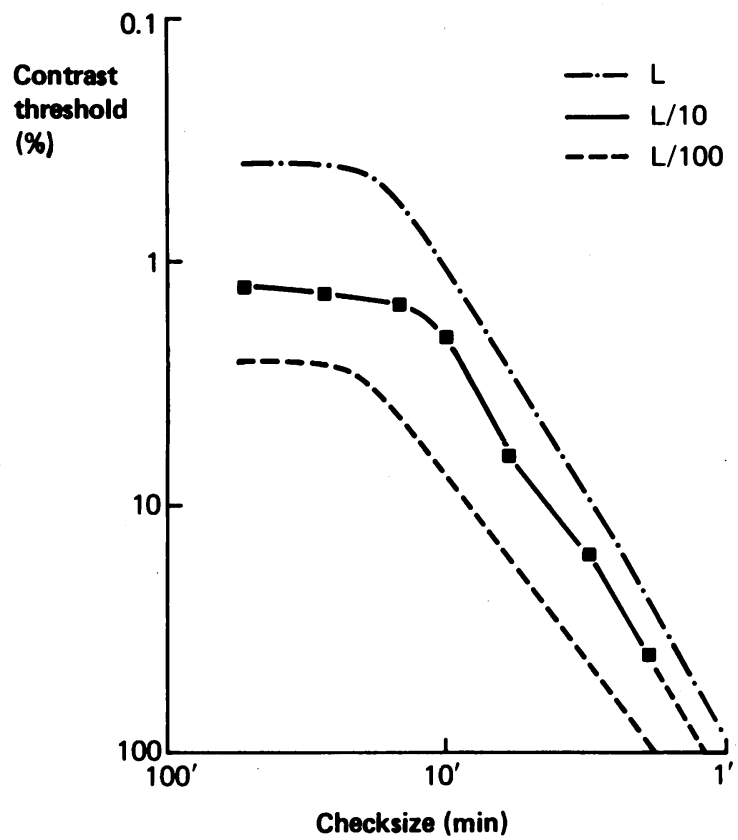

Fig. 7 The effect of mean luminance on the VCSF.

years previously and since then had complained of persistent headaches and reduced visual acuity for both distance and near. Unaided acuities were $6 / 36$ in each eye. Retinoscopy revealed only a small hypermetropic error (+0.50 DS) in each eye and both fundi were entirely healthy. Visual fields showed a spiral contraction. A clinical diagnosis of hysterical amblyopia was made and VECPs were recorded in order to substantiate the diagnosis. Fig. 12 indicates that his contrast sensitivity function is in good general

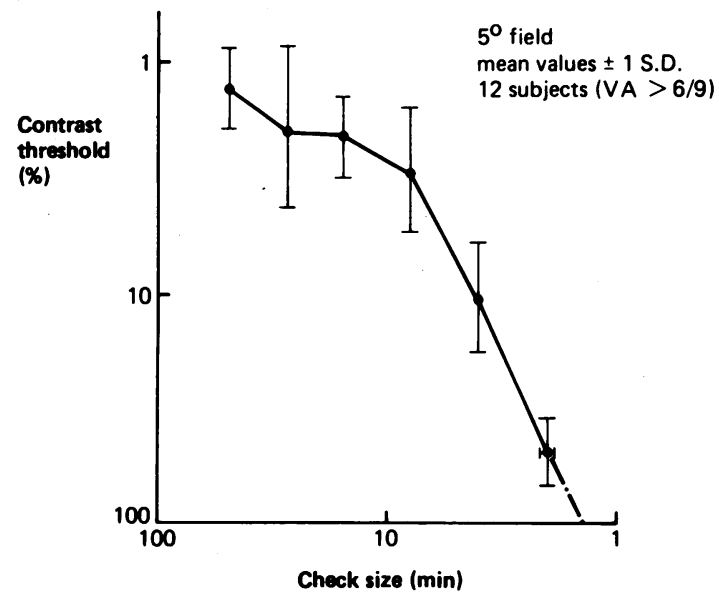

Fig. 8 Mean curve for a group of 12 normal subjects.

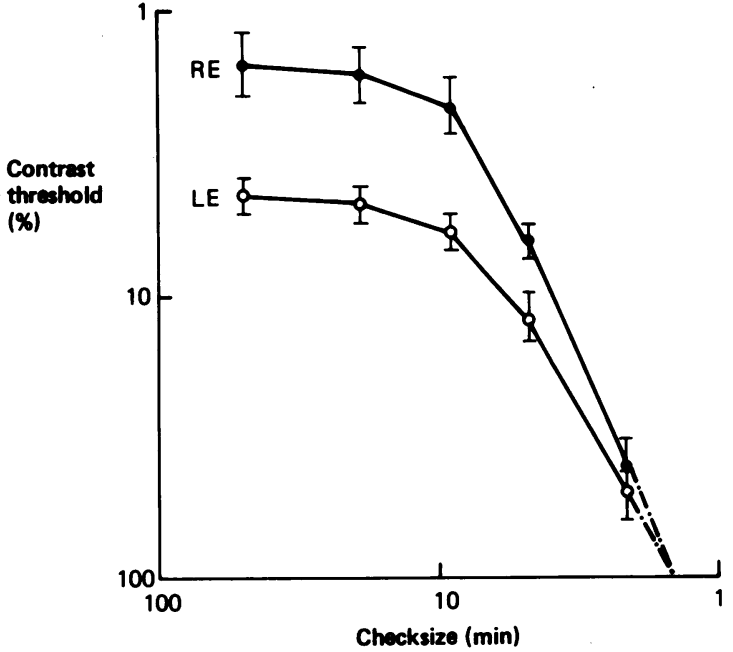

Fig. 9 VCSFs of a patient with early chronic glaucoma of the left eye.

agreement with the mean curve for our group of normal persons (broken line). The most significant deviation occurs at the top end of the curve where extrapolation predicted a slightly reduced acuity $(\simeq 6 / 9)$, which was nevertheless much better than that arrived at by conventional Snellen chart testing. Obviously in such situations as this the technique has considerable value in aiding difficult clinical assessment where there may be profound medicolegal consequences.

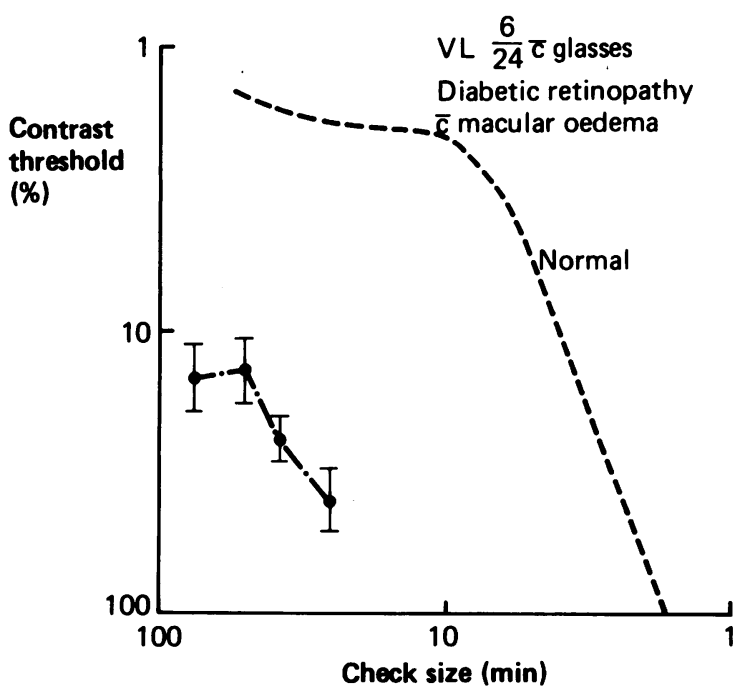

Fig. 10 VCSF of the left eye of a patient with diabetic retinopathy and associated macula oedema. 


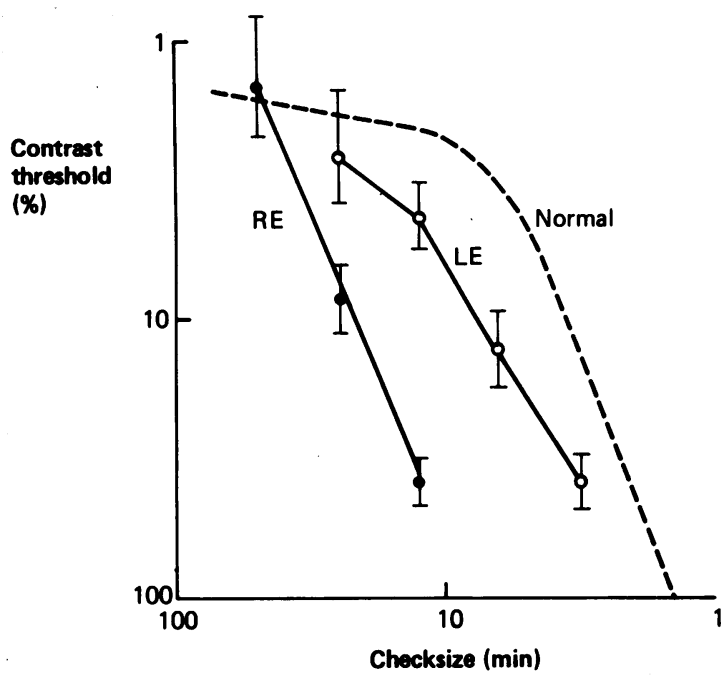

Fig. 11 VCSFs of a patient with a right macular hole.

Strabismic amblyopia. A 28-year-old patient was referred by his own optician because of subnormal acuity in the right eye. On questioning he admitted to having had poor vision in this eye for as long as he could remember. Visual acuity in the right eye could not be improved beyond 6/12 whereas that in the left eye corrected to $6 / 5$. He had a right microstrabismus with eccentric fixation, and a diagnosis of strabismic amblyopia was made on clinical grounds. The contrast

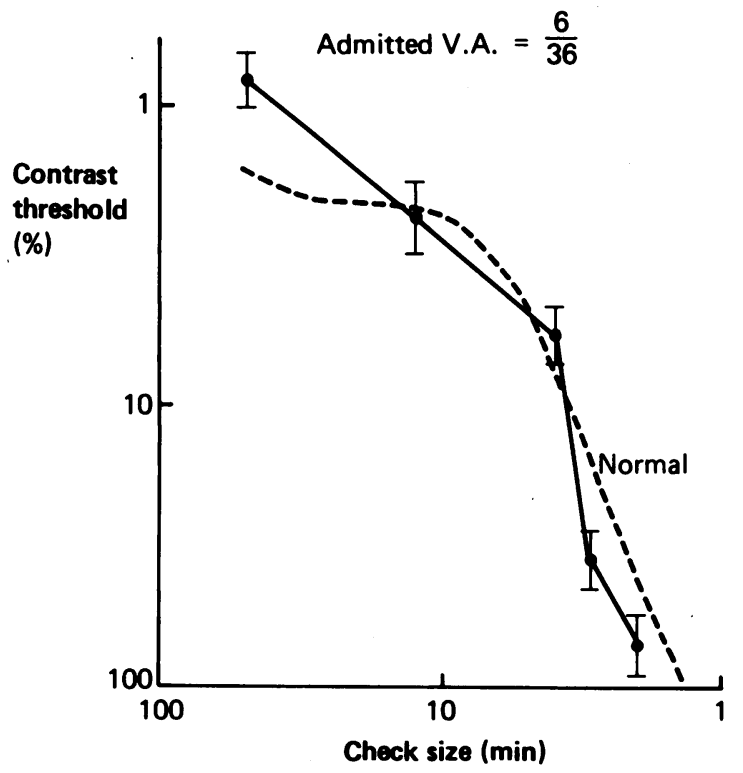

Fig. 12 VCSF of a patient with hysterical amblyopia.

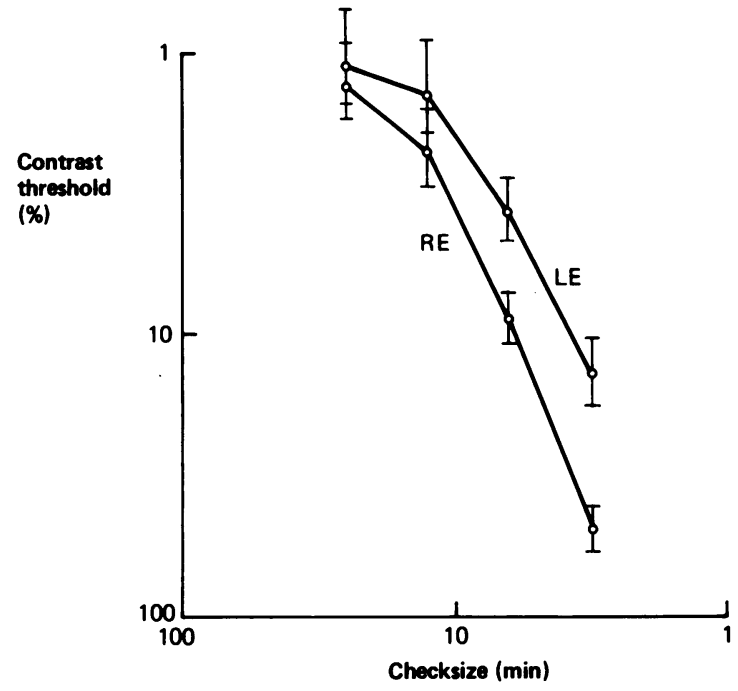

Fig. 13 VCSFs of a subject with strabismic amblyopia of the right eye.

sensitivity functions for each eye are illustrated in Fig. 13. The RE curve shows that sensitivity to small check sizes was impaired, but to large check sizes it was preserved (note that the patient was optimally refracted). Similar effects have been observed by other workers using subjective techniques, and indeed it has been shown that the type of amblyopia -i.e., strabismic, deprivation, or anisometropiccan result in subtle differences in the shape of the contrast sensitivity curve of the affected eye..$^{71} 182228$

These cases are illustrative. The technique has been applied to other disorders in which contrast sensitivity is known to be affected-e.g., macular degeneration, ${ }^{91426}$ retinitis pigmentosa, ${ }^{2024}$ and demyelinating optic nerve disease..$^{811232}$ The method has, however, one significant drawback with regard to its application in the clinical environment-the length of the test. To obtain a contrast sensitivity curve consisting of five points on each eye requires a measurement time of approximately two hours. This is clearly impracticable for a routine test, but the problem can be overcome in most of the examples described by careful rationalisation of the procedure. In the type of cases discussed previously the provisional diagnosis will give a clue to whether central, peripheral, or global visual function is likely to be impaired. Contrast sensitivity assessment can then help describe and quantify the form and degree of such impairment. For example, if a degradation in contrast sensitivity to large check sizes is anticipated (as for example in early chronic glaucoma), the experiment can be performed with one check size only, and comparisons made with either the normal eye or the normal 


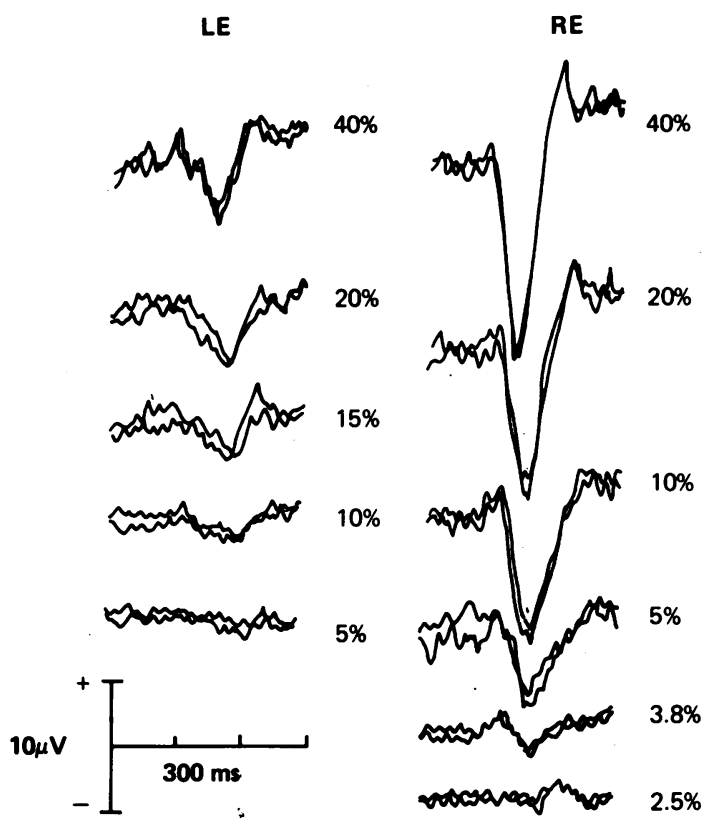

Fig. 14 VECPs to 19 min checks and at a series of contrasts from the patient with early chronic glaucoma of the left eye (Fig. 9).

range. ${ }^{65}$ The VECPs elicited from the patient with apparent uniocular chronic glaucoma (with checks of $19^{\prime}$ subtense, see Fig. 9), and the amplitude versus contrast graphs drawn from these data, are shown respectively in Figs. 14 and 15 . The differences in amplitude and threshold values in the two eyes are clearly seen. However, it is important to note the additional suprathreshold information which is elicited-i.e., the slope of the amplitude versus contrast plot. A difference of nearly a factor of 2 is observed between the two eyes. It would appear that this represents a reduction in 'gain' of the spatial contrast mechanism responsible for the processing of elements of this subtense. It is not an artefact produced by optical blurring, because image degradation of this type leads only to reductions in sensitivity (i.e. increased threshold), with no alteration to the slope of the graph. An experiment to confirm this finding was performed on the normal myope tested previously (Fig. 6), and the results are illustrated in Fig. 16. Threshold is significantly reduced by correction (note the check size), but the slope is essentially unaffected.

It is also possible to have the situation where contrast sensitivity is not measurably reduced but suprathreshold response is (Fig. 17). These data were obtained from the patient with strabismic amblyopia described previously. No significant difference in threshold is observed when the amblyopic eye and the normal eye are compared, but for checks of $25^{\prime}$ a significant difference in slope is clearly evident (in this case greater than a factor of 3 ). Thus it is likely that if contrast sensitivity alone were taken as the only measure, reductions in suprathreshold function could go unrevealed, especially where psychophysical methods were adopted to obtain the VCSF.

This rationalised approach has been particularly useful where serial measurements of contrast sensitivity were required to assess objectively the improvement in visual function after trauma. A patient who received a blunt injury to the right eye during a car accident was found to have macular oedema and slightly reduced visual acuity (6/12 RE, 6/9 LE). Contrast sensitivity (with $25^{\prime}$ checks) was determined on four occasions following the injury and the details are shown in Table 1. Visual acuities
Fig. 15 CI-CII amplitude versus contrast from the data of Fig. 14.

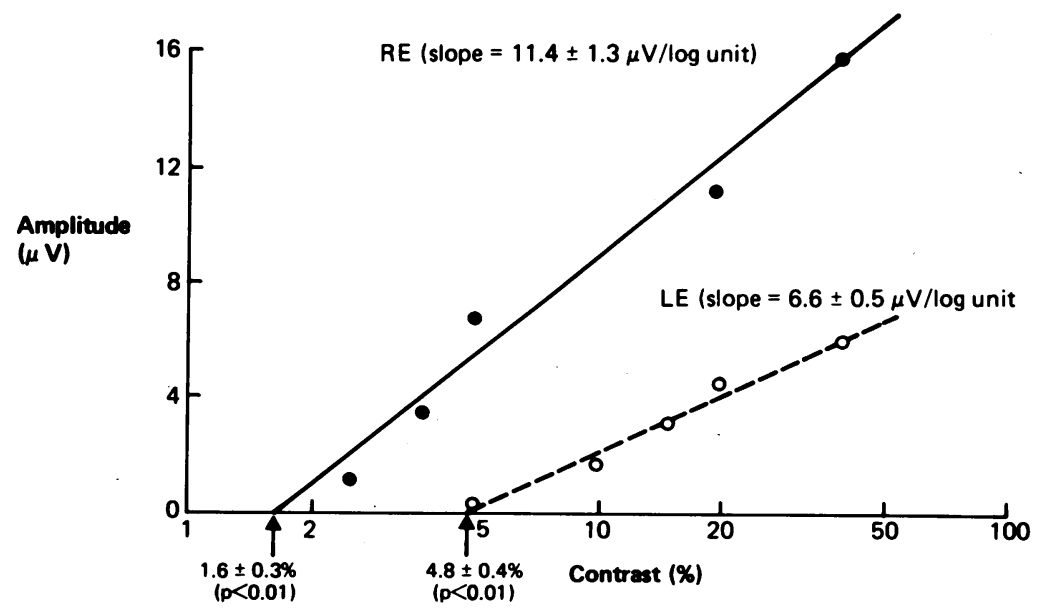


Table 1 Serial measurements on a patient with macular oedema in the right eye

\begin{tabular}{|c|c|c|c|c|}
\hline \multirow{2}{*}{$\begin{array}{l}\text { Period } \\
\text { after } \\
\text { injury }\end{array}$} & \multicolumn{2}{|c|}{ Visual acuity } & \multicolumn{2}{|c|}{ Contrast threshold (\%) } \\
\hline & $R E$ & $L E$ & $R E$ & $L E$ \\
\hline 3 weeks & $6 / 12$ & $6 / 9$ & $11 \cdot 2 \pm 1.4(\mathrm{p}<0.02)$ & $2.7 \pm 0.7(p<0.01)$ \\
\hline 7 weeks & $"$ & $"$ & $9.4 \pm 0.6(p<0.001)$ & $2.8 \pm 0.6(p<0.01)$ \\
\hline 12 weeks & $"$ & $"$ & $4 \cdot 1 \pm 0.2(p<0.001)$ & $2.8 \pm 0.4(p<0.01)$ \\
\hline 17 weeks & $"$ & $"$ & $3.3 \pm 0.7(p<0.01)$ & $2.5 \pm 0.1(\mathrm{p}<0.001)$ \\
\hline
\end{tabular}

were measured at the same time, and these are included for comparison. RE acuity did not change significantly during the measurement period, but contrast sensitivity showed a progressive improvement in keeping with the ophthalmoscopically observed reduction in macular oedema and the subjective improvement in 'vision' observed by the patient. Of particular importance is the consistency of the 'control' data obtained from the unaffected left eye during the investigation period.

\section{Discussion}

\section{STIMULUS PARAMETERS}

When evoked potential techniques are used to determine visual contrast sensitivity function, it is of utmost importance to consider carefully the type of stimulus and its physical parameters, since these will influence both the form of the VECP, and the information contained in it with respect to contrast processing.

Gratings versus checkerboards. Gratings have been used in both psychophysical and many electrophysiological approaches, as they open up the possibility of investigating visual function using modulation transfer functions (i.e., the VCSF), and Fourier analysis techniques in the spatial frequency domain. ${ }^{72-75}$ Their use is further reinforced by the experimental evidence suggesting that spatial processing 'channels', sensitive to discrete stimulus sizes and orientations, exist in the visual system. ${ }^{154676-78}$ The checkerboard has, however, been shown to be a more suitable stimulus, as it more effectively excites the retinal receptive fields which are thought to be approximately circular in shape. ${ }^{5254}$ In recent years the existence of a subfunctional element of spatial contrast processing called the 'edge' or 'contour specific' mechanism has been postulated..$^{55679}$ In these studies experiment has confirmed that pattern generated evoked potentials are sensitive not only to the contrast between spatial elements but to the characteristics of the border or 'edge' separating them. The checkerboard, of course, has twice as many edges as the grating (if one assumes a square wave luminance profile) for an equivalent field size. The checkerboard should therefore elicit larger amplitude responses, and indeed this has been verified by experiment. ${ }^{56}$

Pattern reversal versus onset-offset. Overall luminance changes must be avoided if responses are to be obtained to contrast only. ${ }^{5280}$ Thus the choice of pattern modulation is limited to either 'pattern reversal' or 'onset-offset'. In the former the luminance of the checks is modulated in counterphase, and in the latter the luminance modulation is so arranged to present the pattern for a predetermined period and then return to a blank screen of the same mean luminance level. Both forms are widely used in visual electrophysiology. However, in the context of contrast sensitivity determination the onset-offset stimulus is more suitable, since the potentials evoked are purer contrast generated responses, whereas

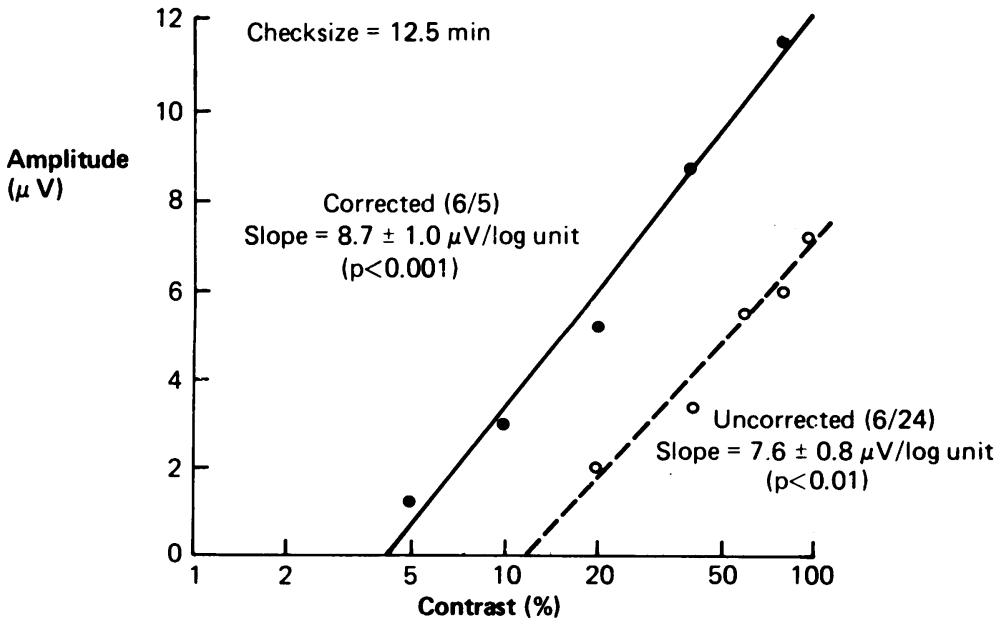

Fig. 16 CI-CII amplitude versus contrast for the previously described myope (Fig. 6) showing the effect of correction. 
Fig. 17 CI-CII amplitude versus contrast data from the patient with strabismic amblyopia (Fig. 13).

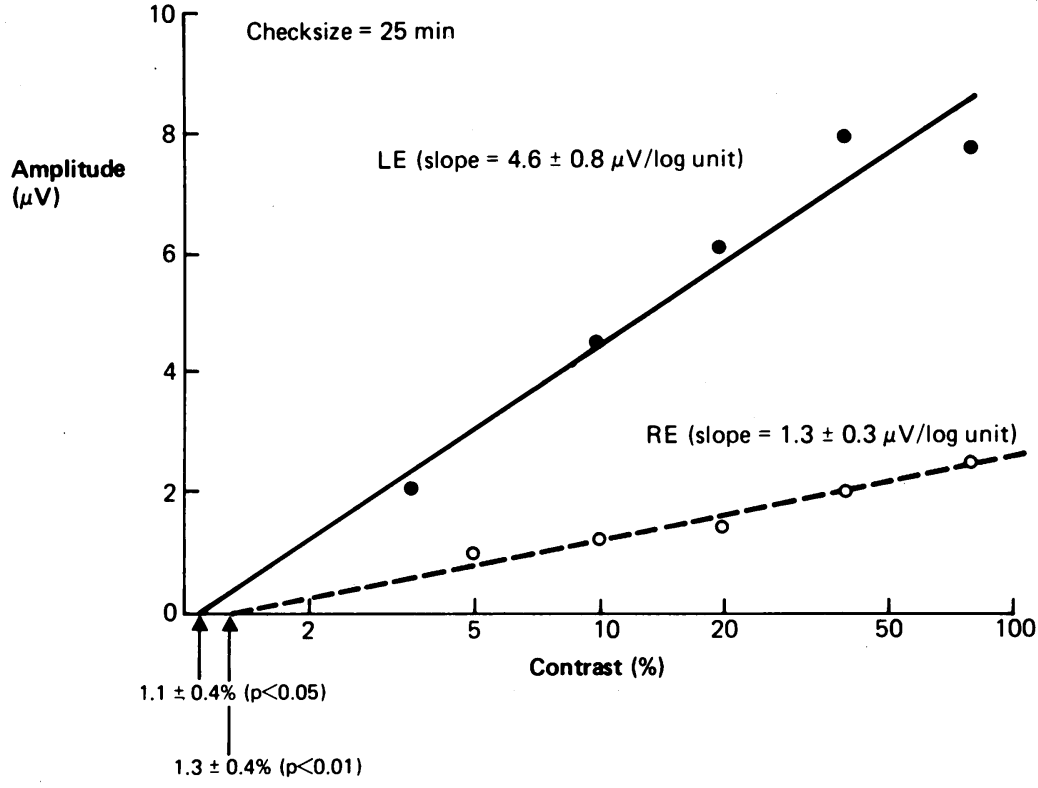

those to pattern reversal are known to be more contaminated by luminance components. 53577981 In addition the edge specific mechanism is adapted out in the pattern reversal stimulus, as the pattern is always present. This can be observed if a pattern reversing checkerboard is fixated for more than half a minute or so since, on removal of the pattern, a dark grid is perceived which gradually fades. A further phenomenon that is observed with pattern reversal is the apparent movement on each reversal. This would suggest that this mode possibly stimulates the 'channel' concerned with movement perception, ${ }^{82}$ although it has been observed that characteristic VECPs to pattern reversal occur irrespective of whether an illusory motion is perceived.$^{83}$ This effect is not observed with onset-offset modulation.

The characteristic form of responses generated by the two types of stimuli are very different. At low stimulus frequencies the pattern reversal VECP shows a positive, monotonic component at a latency of about $100 \mathrm{~ms}$ (the so-called 'P100' component), whereas that to the pattern in onset-offset mode shows the prominent negative component CII, which has similar latency, preceded by the positive component CI. Their amplitude characteristics in response to changes in contrast are not similar (Fig. 18). The pattern reversal responses appear to reach a saturation level at about $20 \%$ contrast (note: mean luminance $=10 \mathrm{Cd} \mathrm{M}^{-2}$ ) and no further increase is observed (there may even be a falling off at high contrast, though the reason for this is unclear); in contradistinction the onset-offset responses increase proportionately with log contrast. As has already been stated, these responses too will eventually reach saturation, but at higher mean luminance levels. ${ }^{52}$

The site of origin and processing of the potentials to the two modes is also possibly different, as their distribution across the scalp is so disparate and is particularly emphasised when upper or lower half fields (or segments thereof) are stimulated. ${ }^{7984}$ Indeed in our own laboratory we have observed in some patients with tapetoretinal degeneration grossly attenuated pattern reversal VECPs but large amplitude onset-offset responses, all other conditions, apart from pattern modulation, being identical. ${ }^{63} \mathrm{On}$ the other hand the patient tested previously with strabismic amblyopia had large amplitude pattern reversal responses which were not much different from those of the normal eye, but grossly attenuated onset-offset VECPs in the affected eye as compared with the normal eye. (Note: implicit in these observations is the assumption that a consistent electrode montage is maintained. Variation will alter the form and amplitude of the VECPs due to the aforementioned topographical effects.)

The maturation of these responses is also dissimilar. The characteristic pattern reversal VECP with P100 component is observed in children, but the fully formed onset-offset VECP with CI-CII components is often not seen till puberty ${ }^{85}$ (and our own unpublished observations).

Apart from the last mentioned situation, where it may not be possible to obtain characteristic onsetoffset responses, experimental evidence points to the 


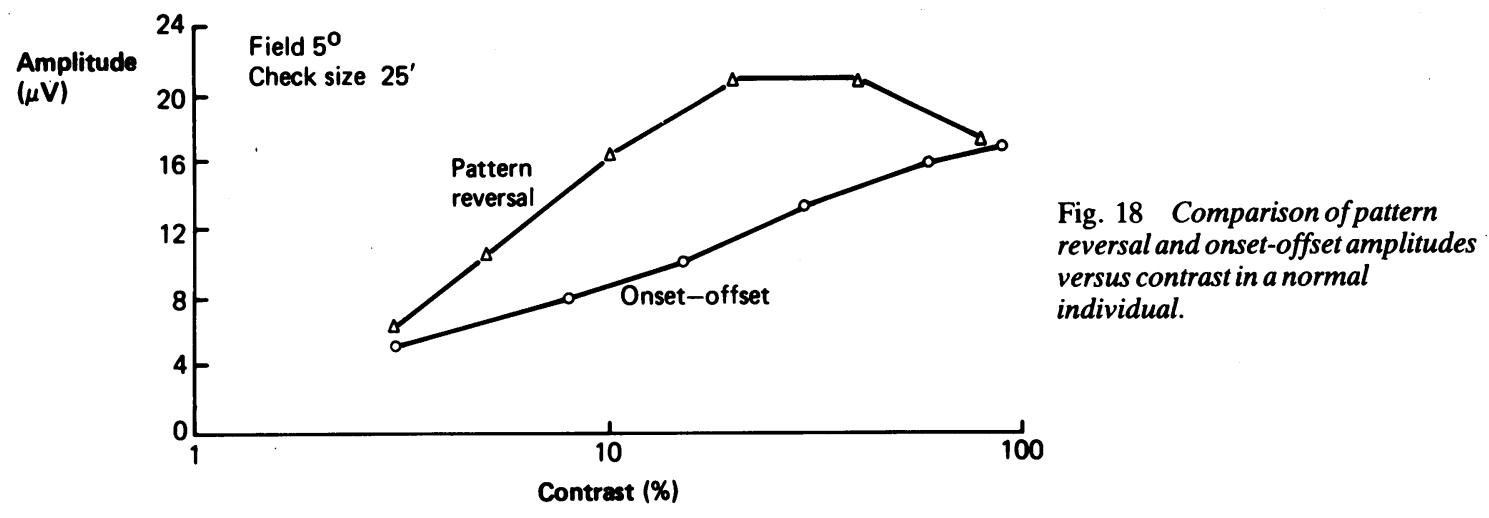

onset-offset checkerboard as the stimulus of choice. The selection of $40 \mathrm{~ms}$ pattern duration is made because it ensures complete integration of the pattern on the retina. ${ }^{52}$

Stimulus Frequency. The waveform of the VECP is significantly affected by stimulus frequency. At high frequencies $(\geqslant 8-10$ reversals/presentations per second) a 'quasisinusoidal' response is elicited (a socalled 'steady-state' response $\left.{ }^{86}\right)$. When lower frequencies are used, however, the discrete components previously mentioned become apparent (so-called 'transient' responses). VECPs behave differently in these two temporal frequency bands, ${ }^{57}$ and the conclusions derived from one are not necessarily relevant to the other. Pattern reversal responses can be elicited up to about 25 reversals per second but onset-offset responses-and in particular the CII component - are considerably attenuated due to pre-exposure adaptation if the interstimulus interval does not exceed the pattern duration by about a factor of $10 .{ }^{7987}$ This places an upper constraint on repetition rate of about 2 per second. Lower stimulus frequencies were not used as they increase the length of the test unacceptably.

Luminance. The mean luminance of the TV screen was adjusted to be a low photopic level of $10 \mathrm{Cd} \mathrm{m}^{-2}$, as high luminance can introduce saturation effects in VECPs. In fact Spekreijse et al..$^{52}$ have observed the phenomenon at contrasts as low as $20 \%$ and check sizes of $20^{\prime}$ upwards. Furthermore, TV tubes are notoriously non-linear devices and contrast/brightness controls are not independent (these characteristics are worsened at high intensity levels, with the unacceptable consequence that, as contrast is increased, so too is mean luminance). Operating at lower luminance minimises these effects.

Range of check size. Those familiar with psychophysical methods of obtaining VCSF will observe that the range of check subtenses within which the function is determined ( $2 \mathrm{~min}$ to $50 \mathrm{~min}$, 'equivalent' to grating frequencies of 11 to 0.4 cycles per degree), is smaller than that usually tested ( 30 to $0 \cdot 1$ cycles per degree). The reason for this is that checks of subtense $>25$ min produce VECPs progressively more contaminated by luminance components, ${ }^{48}$ and checks of subtense $<2 \mathrm{~min}$ are difficult to generate due to TV phosphor limitations, together with acceptable patient-monitor distance/field size constraints. In addition, the evoked potentials to such small checks have very low amplitudes (usually $\leqslant 1 \mu \mathrm{V}$ ), and are virtually impossible to resolve within an average which consists of a reasonable number of epochs $(\leqslant 512)$.

Field size. The selection of field size is important in that the VCSF will be modified, particularly to large check sizes, if only the central $5^{\circ}$ is stimulated. This will not matter if macular function is the purview of the test, but if more peripheral function is being investigated, as in chronic glaucoma for instance, then a larger field size is obviously required (in our case $17^{\circ} \times 14^{\circ}$ ).

\section{CLINICAL APPLICATION}

The construction of a contrast sensitivity curve, under standardised conditions, describes and quantifies the 'envelope of vision' of the patient and is therefore relevant as an aid to diagnosis and subsequent assessment. Disorders affecting contrast processing mechanisms in the retina or higher visual pathways will be reflected in modifications to the shape of the curve which indicate either partial, or total reductions in sensitivity. Indeed the type of attenuation can be characteristic of the disease process being studied. It is of profound clinical importance to be able to interpret in meaningful scientific terms symptoms which the patient may find difficult to explain-for example, situations where good visual acuity is preserved, but contrast discrimination to large objects (which should therefore be easily resolveable) is diminished. Thus, as has already been stated, the 
case made by Arden and others in recent years, and ourselves now, for the routine use of contrast sensitivity measurement at some stage in the clinical assessment, is very convincing. In all methods of contrast sensitivity determination the results must be interpreted with care and due regard paid to the presence of any opacities in the media, since these will of themselves cause a reduction in contrast sensitivity. Extreme miosis or mydriasis will alter overall retinal illumination, and this too will affect contrast sensitivity. It is therefore important to measure pupillary diameter (under test conditions), particularly where monocular stimulation is used and interocular comparisons are being made. Any differences should then be allowed for in the interpretation of the results.

Finally, we have to consider which methòd of determining contrast sensitivity to adopt, as both have advantages and disadvantages. The main problem with the psychophysical approach is that it is not readily applicable to patients who are either confused or uncooperative. An additional disadvantage is that very important suprathreshold data cannot be obtained. On the other hand a simple psychophysical method involving the use of a series of contrast grating plates, such as Arden's, has proved useful with co-operative patients in the clinical environment.

The electrophysiological technique is an alternative, objective method which requires only that the patient maintain good fixation on the TV monitor. Threshold and suprathreshold data are elicited, and, as a bonus, the evoked potentials give further information on the state of optic nerve conduction, which in many of the cases cited is also affected. There are of course drawbacks with this method. Acquisition of the data to establish a complete contrast sensitivity curve requires an unacceptably long test period. As described, the test can be rationalised to save time and only relevant data to the condition being investigated acquired, thus reducing the length of the test to a more realistic 20-25 minutes. Other problems arise which, although common to all evoked potential techniques, must be approached with care and necessitate good EEG practice. ${ }^{88}{ }^{89} \mathrm{In}$ particular, responses contaminated by noise (usually $50 \mathrm{~Hz}$ ) (TV stimulators can introduce special problems in this respect ${ }^{90}$ ) or by alpha cortical activity must be eliminated. However, as long as these factors are taken into consideration and a careful and consistent approach to the analysis of data is made, we have found that the electrophysiological determination of contrast sensitivity function is a practical proposition within the confines of an electrophysiology laboratory. It provides clinically valuable information regarding threshold and suprathreshold function and gives further insight into the nature of the visual disability.

The authors wish to express their appreciation to Ms Karen Copeland, medical physics technician, who skilfully elicited a large proportion of the data presented in this paper.

\section{References}

1 Campbell FW, Green DJ. Optical and retinal factors affecting visual resolution. J Physiol (Lond) 1965 ; 181: 576-93.

2 Robson JG. Spatial and temporal contrast sensitivity functions of the visual system. J Opt Soc Am 1966; 56: 1141-2.

3 Bodis-Wollner I. Visual acuity and contrast sensitivity in patients with cerebral lesions. Science 1972; 178: 769-71.

4 Bodis-Wollner I, Hendley CD, Kulikowski JJ. Psychophysical and electrophysiological responses to the modulation of contrast of a grating pattern. Perception 1972; 1: 341-9.

5 Campbell FW. The transmission of spatial information through the visual system. In: Schmidt FO, Wooden FG, eds. The neurosciences; third study programme. Cambridge, Massachusetts: MIT Press, 1974: 95-103.

6 Bodis-Wollner I, Diamond SP. The measurement of spatial contrast sensitivity in cases of blurred vision associated with cerebral lesions. Brain 1976; 99: 695-710.

7 Hess RF, Howell ER. The threshold contrast sensitivity function in strabismic amblyopia; evidence for a two type classification. Vison Res 1977; 17: 1049-55.

8 Regan D, Silver R, Murray TJ. Visual acuity and contrast sensitivity in multiple sclerosis; hidden visual loss. Brain 1977; 100: $563-79$.

9 Sjöstrand J, Frisen L. Contrast sensitivity in macular disease. Acta Ophthalmol (Kbh) 1977; 55: 507-14.

10 Wolkstein M, Atkin A, Bodis-Wollner I. Grating acuity in two sisters with tapeto-retinal degeneration. Doc Ophthalmol 1977; 13: 41-6.

11 Bodis-Wollner I, Hendley CD, Mylin AT, Thornton J. Visual evoked potentials and the visuogram in multiple sclerosis. Neurology 1978; 5: 40.

12 Frisen L, Sjöstrand J. Contrast sensitivity in optic neuritis. Doc Ophthalmol Proc Ser 1978; 17: 165-74.

13 Arundale $K$. An investigation into the variation of human contrast sensitivity with age and ocular pathology. $\mathrm{Br} J \mathrm{Oph}$ thalmol 1978; 62: 213-15.

14 Sjöstrand J. Contrast sensitivity in macular disease using a small field and a large field TV system. Acta Ophthalmol (Kbh) 1979; 57: $832-46$.

15 Campbell FW. Recent attempts to link psychophysics with neurophysiology in vision research. Trans Ophthalmol Soc UK 1979; 99: 326-32.

16 Atkin A, Bodis-Wollner I, Wolkstein M, Moss A, Podos SM. Abnormalities of central contrast sensitivity in glaucoma. Am I Ophthalmol 1979; 88: 205-11.

17 Hess RF. Contrast sensitivity assessment of functional amblyopia in humans. Trans Ophthalmol Soc UK 1979; 99: 391-7.

18 Levi DM, Harwerth RS. Contrast sensitivity in amblyopia due to stimulus deprivation. Br J Ophthalmol 1980; 64: 15-20.

19 Bodis-Wollner I, Camisa JM. Contrast sensitivity measurement in clinical diagnosis. In: Lessell S, Van Dalen JTW, eds. Neuroophthalmology. Oxford: Excerpta Medica, 1980: 11: 373-401.

20 Hyvarinen L, Rovamo J, Laurinen P, Peltomes A. Contrast sensitivity in retinitis pigmentosa. Acta Ophthalmol (Kbh) 1981; 59: 763-73.

21 Lundh B, Lennestrand G. Eccentric contrast sensitivity loss in chronic glaucoma. Acta Ophthalmol (Kbh) 1981; 59: 21-4.

22 Sjöstrand J. Contrast sensitivity in strabismic and anisometropic amblyopia. A study of the effect of treatment. Acta Ophthalmol (Kbh) 1981; 59: 25-34. 
23 Woo GC, Dalziel CC. A pilot study of contrast sensitivity assessment of the CAM treatment of amblyopia. Acta Ophthalmol (Kbh) 1981; 59: 35-7.

24 Kayazawa F, Yamamoto T, Itoi M. Contrast sensitivity measurement in retinal diseases to laser generated sinusoidal grating. Acta Ophthalmol (Kbh) 1982; 60: 511-24.

25 Hyvärinen L, Laurinen P, Rovamo J. Contrast sensitivity in evaluation of visual impairment due to diabetes. Acta Ophthalmol (Kbh) 1983; 61: 94-101.

26 Hyvärinen L, Laurinen P, Rovamo J. Contrast sensitivity in evaluation of visual impairment due to macular degeneration and optic nerve lesions. Acta Ophthalmol (Kbh) 1983; 61: 161-70.

27 Lundh B, Lennerstrand G, Derefeldt G. Central and peripheral normal contrast sensitivity for static and dynamic sinusoidal gratings. Acta Ophthalmol (Kbh) 1983; 61: 171-82.

28 Lundh B, Lennerstrand G. Effects of amblyopia therapy on contrast sensitivity as reflected in the visuogram. Acta Ophthalmol (Kbh) 1983; 61: 431-46.

29 Arden GB. The importance of measuring contrast sensitivity in cases of visual disturbance. Br J Ophthalmol 1978; 62: 198-209.

30 Arden GB. Visual loss in patients with normal visual acuity. Trans Ophthalmol Soc UK 1978; 98: 219-23.

31 Arden GB, Jacobson JJ. A simple grating test of contrast sensitivity. Preliminary results indicate value in screening for glaucoma. Invest Ophthalmol Visual Sci 1978; 17: 23-32.

32 Arden GB, Gucukoglu AG. Grating test of contrast sensitivity in patients with retrobulbar neuritis. Arch Ophthalmol 1978; 96: $1626-9$.

33 Minassian DC, Jones BR, Zargarizaden A. The Arden grating test of visual function: a preliminary study of its practicability and application in a rural community in North-West Iran. $\mathrm{BrJ} \mathrm{Oph}$ thalmol 1978; 62: 210-2.

34 Skalka HW. Effect of age on Arden grating acuity. $\mathrm{Br} J \mathrm{Oph}$ thalmol 1980; 64: 21-3.

35 Skalka HW. Comparison of Snellen acuity, VER acuity, and Arden grating scores in macular and optic nerve diseases. $\mathrm{Br} \mathrm{J}$ Ophthalmol 1980; 64: 24-9.

36 Canavan Y, Archer DB. Loss of contrast sensitivity following contusional eye injury. BrJ Ophthalmol 1980; 64: 613-7.

37 Sokol S, Domar A, Moskowitz A. Utility of the Arden grating test in glaucoma screening: high false positive rate in normals over 50 years of age. Invest Ophthalmol Visual Sci 1980; 19: 1529-33.

38 Vaegan. The clinical value of printed contrast sensitivity tests. $J$ Physiol (Lond) 1980; 300: 76P.

39 Editorial. Spatial contrast sensitivity revisited. Br J Ophthalmol 1981; 65: 513-4.

40 Hitchings RA, Powell DJ, Arden GB, Carter RM. Contrast sensitivity gratings in glaucoma family screening. $\mathrm{Br} \mathrm{J} \mathrm{Oph-}$ thalmol 1981; 65: 515-7.

41 Singh H, Cooper RL, Alder VA, Crawford GJ, Terrell A, Constable IJ. The Arden grating acuity: effect of age and optical factors in the normal patient, with prediction of the false negative rate in screening for glaucoma. Br JOphthalmol 1981; 65: 518-24.

42 Stamper RL, Hsu-Winges C, Sopher M. Arden contrast sensitivity testing in glaucoma. Arch Ophthalmol 1982; 100: 947-50.

43 Marsh RJ, Ford SM, Rabb MF, Hayes RJ, Serjeant GR. Macular vasculature, visual acuity, and irreversibly sickled cells in homozygous sickle cell disease. Br J Ophthalmol 1982; 66: 155-60.

44 Vaegan, Halliday BL. A forced-choice test improves clinical contrast sensitivity testing. BrJ Ophthalmol 1982; 66: 477-91.

45 Ghafour IM, Foulds WS, Allan D, McClure E. Contrast sensitivity in diabetic subjects with and without retinopathy. $\mathrm{Br} J$ Ophthalmol 1982; 66: 492-95.

46 Campbell FW, Maffei L. Electrophysiological evidence for the existence of orientation and size detectors in the human visual system. J Physiol (Lond) 1970; 207: 635-52.

47 Campbell FW, Kulikowski JJ. The visual evoked potential as a function of contrast of a grating pattern. $J$ Physiol (London) 1972; 222: 345-56.
48 Spekreijse $\mathrm{H}$. Analysis of EEG responses to diffuse and patterned light in the human. Thesis. The Hague: D W Junk, 1966.

49 Jeffries DA, Axford JG. Source locations of pattern-specific components of human visual evoked potentials. I. Component of striate cortical origin. Exp Brain Res 1972; 16: 1-21.

50 Jeffries DA, Axford JG. Source locations of pattern-specific components of human visual evoked potentials. II. Component of striate cortical origin. Exp Brain Res 1972; 16: 22-40.

51 Regan D. Evoked potentials in psychology, sensory physiology and clinical medicine. London: Chapman and Hall, 1972.

52 Spekreijse H, Van der Tweel L, Zuidema T. Contrast evoked responses in man. Vision Res 1973; 13: 1577-601.

53 Regan D. Evoked potentials specific to spatial patterns of luminance and colour. Vision Res 1973; 13: 2381-401.

54 Spekreijse H, Estevez O, Reits D. Visual evoked potentials and the physiological analysis of visual processes in man. In: Desmedt $\mathrm{JE}$, ed. Visual evoked potentials in man: new developments. Oxford: Clarendon Press, 1977: 16-89.

55 Van der Tweel LH. Pattern evoked potentials: facts and considerations. In: Tazawa Y, ed. Proceedings of the 16th ISCEV Symposium Morioka. Jpn J Ophthalmol 1979; 27-46.

56 Spekreijse H. Pattern evoked potentials; principles, methodology and phenomenology. In: Barber C, ed. Evoked potentials. Lancaster: MTP Press, 1980: 55-74.

57 Regan D. Evoked potential studies of visual perception. Can J Psychol 1981; 35: 77-112.

58 Harris L, Atkinson J, Braddick O. Visual contrast sensitivity in a 6-month old infant measured by the evoked potential. Nature 1976; 264: 570-1.

59 Pirchio M, Spinelli D, Fiorentini A, Maffei L. Infant contrast sensitivity evaluated by evoked potential. Brain Res 1978; 141: 179.

60 Atkinson J, Braddick O, French J. Contrast sensitivity of the human neonate measured by the VEP. Invest Ophthalmol Visual Sci 1980; 19: 950-5.

61 Fiorentini A, Pirchio M, Spinelli D. Scotopic contrast sensitivity in infants evaluated by evoked potentials. Invest Ophthalmol Visual Sci 1980; 19: 950-5.

62 Howe JW, Mitchell KW, Robson C. Electrophysiological assessment of visual acuity. Trans Ophthalmol Soc UK 1981; 101: 105-8.

63 Howe JW, Mitchell KW, Robson C. Some clinical experiences using contrast evoked potential techniques in organic and nonorganic visual dysfunction. Doc Ophthalmol Proc Ser 1982; 31: 353-60.

64 Mitchell KW, Howe JW. Assessment of changes in VECP latency and spatial contrast sensitivity in patients with chronic glaucoma and suspected glaucoma. Doc Ophthalmol Ser 1982; 31: 445.

65 Howe JW, Mitchell KW. Visual evoked cortical potential in patients with glaucoma and suspected glaucoma. Res Clin Forums 1982; 4: 41-53.

66 Yamazaki H, Adachi-Usami E, Chiba J. Contrast thresholds of diabetic patients determined by means of the VECP and psychophysical measurements. Acta Ophthalmol (Kbh) 1982; 60: 386-92.

67 Jasper HH. Report of a committee on methods of clinical examination in electroencephalography. Electroencephalogr Clin Neurophysiol 1958; 10: 370-5.

68 Daitch JM, Green DG. Contrast sensitivity of the human peripheral retina. Vision Res 1969; 9: 947-52.

69 Hecht S. Vision II. The nature of the photoreceptor process. In: Murchison C, ed. A handbook of general experimental psychology. Worcester, Md: Clark University Press, 1934: 704-828.

70 Derefedlt G, Lennerstrand G, Lundh B. Age variations in normal human contrast sensitivity. Acta Ophthalmol (Kbh) 1979; 57: 679-90.

71 Sekuler R, Hutman LP. Owesley CJ. Human ageing and spatial vision. Science 1980; 209: 1255-6.

72 Maffei L, Fiorentini A. Processes of synthesis in visual perception. Nature 1972; 240: 479-81. 
73 Maffei L, Fiorentini A. The visual cortex as a spatial frequency analyser. Vision Res 1973; 13: 1255-67.

$74 \mathrm{De}$ Valois KK. Adaptation and the missing fundamental. Invest Ophthalmol Visual Sci 1978; 17 (suppl): 243.

75 Van der Tweel LH, Estevez O, Pijn JPM. Notes on Fourier methods in vision research. Doc Ophthalmol Proc Ser 1983; 37: 439-52.

76 Campbell FW, Cooper GF, Enroth-Cugell C. The spatial selectivity of the visual cells of the cat. J Physiol (Lond) 1969; 203: 223-35.

77. Blakemore C, Campbell FW. On the existence of neurones in the human visual system selectively sensitive to the orientation and size of retinal images. J Physiol (Lond) 1969; 203: 236-60.

78 Smith AT, Jeffreys DA. Size and orientation specificity of transient visual evoked potentials in man. Vision Res 1978; 18: 651-55.

79 Jeffreys DA. The physiological significance of pattern visual evoked potentials. In: Desmedt JE, ed. Visual evoked potentials in man: new developments. Oxford: Clarendon Press, 1977: 134-67.

80 van Lith GHM, Henkes HE, Vijfvinkel-Bruinenga S. Asymmetric pattern evoked responses and stimulus parameters. Doc Ophthalmol Proc Ser 1980; 23: 249-53.

81 Estevez $\mathrm{O}$, Spekreijse $\mathrm{H}$. Relationship between pattern appearance-disappearance and pattern reversal responses. Exp Brain Res 1974; 19: 233-8.

82 Mackay DM, ed. Evoked brain potentials as indicators of sensory information processing. Neurosci Res Program Bull 1969; 7: 3.
83 Van der Tweel LH, Regan D, Spekreijse H. Some aspects of potentials evoked by changes in spatial brightness contrast. 7 th ISCERG Symposium, Istanbul (1969). University of Istanbul: 1971: 1-11.

84 Halliday AM, Barrett G, Halliday E, Michael WF. The topography of the pattern-evoked potential. In: Desmedt JE, ed. Visual evoked potentials in man: new developments. Oxford: Clarendon Press, 1977: 121-33.

85 De Vries-Khoe LH, Spekreijse H. Maturation of luminance and pattern EP's in man. Doc Ophthalmol Proc Ser 1882; 31: 461-75.

86 Regan D. Some characteristics of average steady state and transient responses evoked by modulated light. Electroencephalogr Clin Neurophysiol 1966; 20: 238-48.

87 Barber C, Galloway NR. Adaptation effects in the transient visual evoked potential. In: Lehmann D, Callway E, eds. Human evoked potentials: applications and problems. New York: Plenum Press, 1979: 17-30.

88 Methodology of patterned visual stimulation (report of the Brussels Symposium ad-hoc committee). In: Desmedt JE, ed. Visual evoked potentials in man: new developments. Oxford: Clarendon Press, 1977: 3-15.

89 Report of the Committee on Instrumentation and Procedures in Visual Electrophysiology at the request of the Concilium Ophthalmogicum Universale. Doc Ophthalmol 1981; 1-13.

90 van Lith GHM, van Marle GW, Vijfvinkel-Bruinenga S. Interference of $50 \mathrm{~Hz}$ electrical cortical potentials evoked by TV systems. Br J Ophthalmol 1979; 63: 779-81. 